\title{
Estudios interdisciplinarios en la costa centro-sur de la provincia de Manabí (Ecuador): nuevos enfoques
}

Recherches interdisciplinaires sur la côte méridionale de la province de Manabí (Équateur): nouvelles perspectives

Interdisciplinary Studies in the south-central coast of Manabi Province

(Ecuador): new approaches

Valentina L. Martínez, Yann Graber y Michael S. Harris

\section{OpenEdition \\ Journals}

Edición electrónica

URL: http://journals.openedition.org/bifea/3956

DOI: 10.4000/bifea.3956

ISSN: 2076-5827

Editor

Institut Français d'Études Andines

Edición impresa

Fecha de publicación: 1 diciembre 2006

Paginación: 433-444

ISSN: 0303-7495

Referencia electrónica

Valentina L. Martínez, Yann Graber y Michael S. Harris, « Estudios interdisciplinarios en la costa centro-sur de la provincia de Manabí (Ecuador): nuevos enfoques », Bulletin de l'Institut français d'études andines [En línea], 35 (3) | 2006, Publicado el 01 junio 2007, consultado el 03 diciembre 2020. URL http://journals.openedition.org/bifea/3956 ; DOl : https://doi.org/10.4000/bifea.3956

\section{(c)}

Les contenus du Bulletin de l'Institut français d'études andines sont mis à disposition selon les termes de la licence Creative Commons Attribution - Pas d'Utilisation Commerciale - Pas de Modification 4.0 International. 


\title{
Estudios interdisciplinarios en la costa centro-sur de la provincia de Manabí (Ecuador): nuevos enfoques
}

\author{
Valentina L. Martínez* \\ Yann Graber** \\ Michael S. Harris ${ }^{* * *}$
}

\begin{abstract}
Resumen
Desde el año 2000, Florida Atlantic University está llevando a cabo un estudio interdisciplinario en el área para entender las interacciones complejas entre los grupos humanos y el medio ambiente en el cual se desenvuelven. En este artículo, nosotros reportamos resultados preliminares de nuestra investigación que combina etnografía, arqueología (prospección y excavación) y ciencias naturales. Este enfoque es necesario para poder entender las relaciones co-evolutivas entre varias dimensiones culturales tales como la explotación humana de la naturaleza, formaciones económicas y políticas y organización social. Los resultados finales arrojarán nueva información para explicar patrones de cambio y continuidad local y para hacer contribuciones en la literatura sobre la evolución de sociedades humanas en el Nuevo Mundo.
\end{abstract}

Palabras clave: interdisciplinario, medio ambiente, etnografía, prospección, excavación

\section{Recherches interdisciplinaires sur la côte méridionale de la province de Manabí (Équateur): nouvelles perspectives}

\section{Résumé}

Depuis 2000, l'université de Floride mène une étude interdisciplinaire en vue d'appréhender les interactions complexes entre groupes humains et environnement. Dans cet article, nous présentons les résultats préliminaires de notre recherche qui combine ethnographie, archéologie (prospection

* Departamento de Antropología, Florida Atlantic University (Boca Raton, Florida). E-mail: vmartine@fau.edu

** Universidad de Neuchatel/Latenium, Espace Paul Volga. E-mail: yann.graber@unine.ch

*** Departamento de Antropología, Florida Atlantic University (Boca Raton, Florida). E-mail: mharris@fau.edu 
et fouilles) et sciences naturelles. Cette approche se révèle nécessaire pour la compréhension des relations co-évolutives entre diverses dimensions culturelles comme l'exploitation par I'homme de la nature, les formations politico-économiques et l'organisation sociale. Les conclusions de cette étude devraient éclairer certaines modalités de changement ou, au contraire, de continuité et contribuer, par des publications, aux connaissances sur l'évolution des sociétés humaines dans le Nouveau Monde.

Mots clés : interdisciplinarité, environnement, ethnographie, prospection, archéologie

\title{
Interdisciplinary Studies in the south-central coast of Manabi Province (Ecuador): new approaches
}

\begin{abstract}
Since the year 2000, Florida Atlantic University has been conducting interdisciplinary research to understand the complex interactions between human groups and their environments. In this article, we report preliminary results of our research that combines ethnography, archaeology (survey and excavations), and the natural sciences. This approach is necessary to understand the co-evolutionary relationships among such dimensions as the human exploitation of nature, economic and political formations and social organization. The final results will provide new information to explain the local patterns of change and continuity and contribute to the literature on the evolution of human societies in the New World.
\end{abstract}

Key words: interdisciplinary, environments, ethnography, survey, archaeology

\section{OBJETIVOS DE LA INVESTIGACIÓN}

El área de estudio comprende unos 200 km², desde Puerto López hasta Ayampe, y está compuesta por una serie de comunidades costeras que explotan una variedad de hábitats o ecosistemas en un espacio relativamente pequeño. En la costa centro-sur de la provincia de Manabí (Ecuador), varias investigaciones arqueológicas orientadas a la excavación de sitios específicos revelaron la presencia de depósitos culturales sucesivos. Estas ocupaciones humanas permanentes se iniciaron unos 5000 años atrás y continuaron hasta la llegada de los conquistadores.

En la literatura científica, todavía no se han desarrollado esfuerzos dirigidos a la elaboración de modelos teóricos dinámicos que ayuden a explicar esta rica prehistoria. Al contrario, el récord arqueológico, con la ayuda de análisis cerámicos, ha sido articulado sobre la base de un patrón de cambio y continuidad a través del tiempo (Norton et al., 1983). Investigaciones anteriores tampoco han considerado la presencia de poblaciones actuales en el área; grupos que sugieren una continuidad con el pasado y son fuentes cruciales para la formulación de hipótesis «puente».

A través de un estudio interdisciplinario estamos tratando de entender las interacciones entre poblaciones indígenas y ecosistemas locales desde 5000 años atrás hasta el presente. Aparentemente, los pobladores del área supieron explotar desde la antigüedad los diversos hábitats simultáneamente. Sin embargo, se vislumbran periodos en que los recursos obtenidos en un hábitat adquirieron mayor importancia económica, lo que probablemente ocasionó cambios sociales y políticos en la región. 


\section{MEDIO AMBIENTE: DIVERSOS ECOSISTEMAS O HÁBITATS}

El clima de la región costa del Ecuador depende de las corrientes marinas (Humbolt y El Niño) y de su inversión. Entre los meses de mayo y octubre, la corriente meridional fría del Humbolt afecta las aguas del océano ocasionando la presencia de altos niveles de humedad pero poca precipitación. Entre los meses de diciembre a abril, la corriente de El Niño ocasiona la llegada de masas de aire cálidas y húmedas engendrando un fuerte aumento pluviométrico (Pourrut \& Gómez, 1998: 454).

La costa centro-sur de la provincia de Manabí tiene un clima tropical mega-térmico seco, caracterizado por un régimen pluvial anual que oscila entre 500 y $1000 \mathrm{~mm}$. En esta región, la cordillera de Chongón Colonche baja a la franja litoral determinando, de manera muy sutil, el clima. Una llovizna constante (garúa) se hace presente durante los meses de verano, ocasionada por la condensación de masas de aire oceánicas al ser atrapadas por la cordillera.

La variabilidad en el relieve de la cordillera ha determinado la presencia de varios ecosistemas o hábitats asociados a micro-climas y comunidades bióticas específicas. Nuestras investigaciones sugieren que ambas poblaciones, modernas y prehistóricas, han utilizado los variados ecosistemas en sus prácticas de subsistencia. Estos ecosistemas incluyen: la franja litoral (fig. 1), los valles costeros con un bosque seco (0-50 m.s.n.m.), terrazas interiores con un régimen subtropical (50-80 m.s.n.m.) y las montañas de bosque húmedo (que superan los 80 m.s.n.m.). Adicionalmente, cerca de los ríos se forma también un ecotono o zona ribereña semi-húmeda de transición aprovechada para ciertos cultivos. Hacia la parte oriental de nuestra área de estudio, las cimas de la cordillera alcanzan los 700 metros de altura.

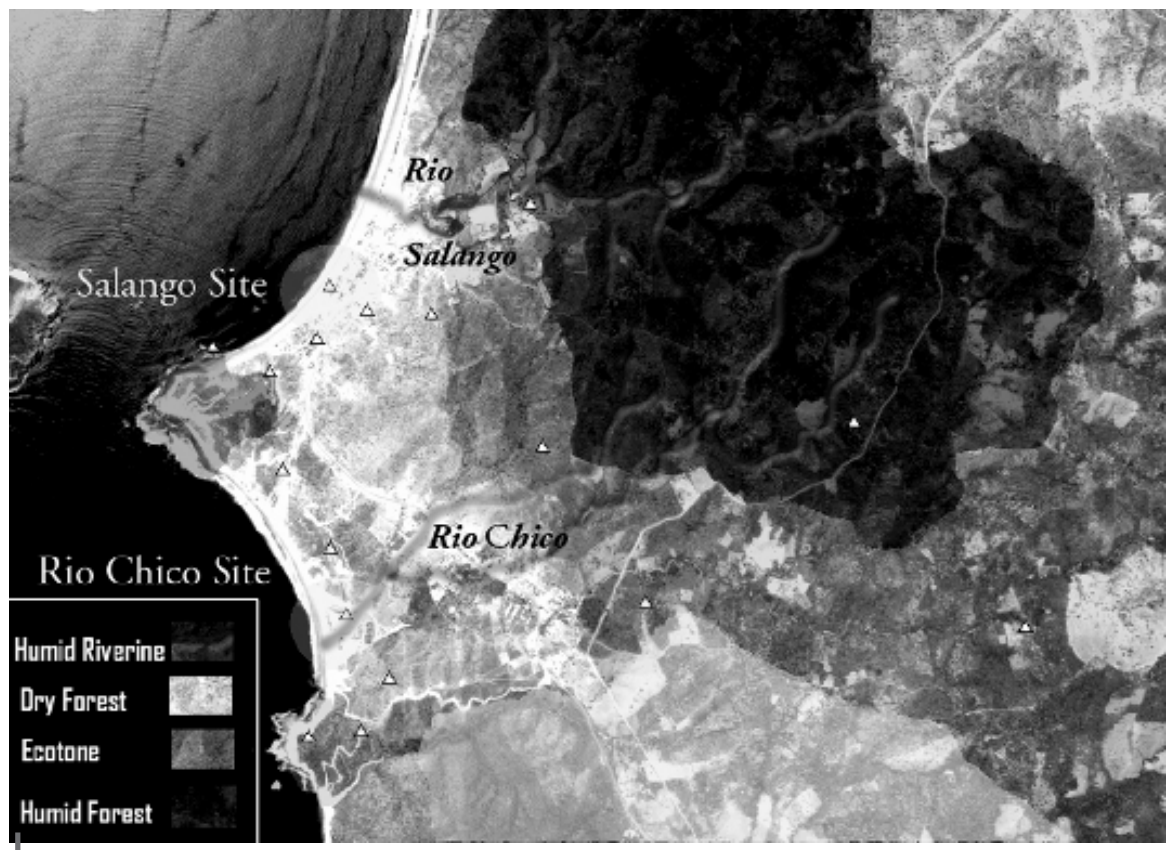

Figura 1 - Los diversos hábitats de la Costa Centro-sur (provincia de Manabí, Ecuador) Mapa elaborado por Charles Roberts 


\section{LA POBLACIÓN ACTUAL: RECURSOS LOCALES, ORGANIZACIÓN SOCIAL Y CONFLICTOS POLÍTICOS}

Gran parte de las comunidades localizadas en la franja litoral se dedican a la pesca, el buceo y la horticultura (Ventura, 1997). En Salango, las dos primeras actividades se realizan en pequeños botes motorizados con redes de arrastre, en botes de fibra de vidrio y canoas de madera. Parte de la recolección marina (pescado, pulpo, calamares, etc.) es inmediatamente distribuida a nivel local entre familiares (nucleares y extendidos), amigos y ancianos. Luego, la mayoría de la pesca es redistribuida en diversas zonas urbanas, especialmente en Guayaquil.

La horticultura se practica en las terrazas interiores o «lomas» (término utilizado localmente) de régimen subtropical que se levantan a lo largo de la costa. La mayoría de grupos domésticos tienen parcelas en las «lomas» donde cultivan yuca, maíz, plátano y frejol. Dicha horticultura se realiza con una tecnología simple a base de machete, sin fertilizantes y sin irrigación. En las montañas de bosque húmedo se practica también una estrategia agroforestal explotando una serie de árboles frutales y madereros para consumo doméstico y del mercado local.

La población de Salango está dividida en dos facciones políticas: La Parroquia y La Comuna. La Comuna es una organización basada en la tenencia y distribución de tierras comunales. Fue creada en los años setenta y está registrada oficialmente en el Ministerio de Agricultura y Ganadería. Su creación fue el resultado de veinte años de lucha por parte de poblaciones rurales para recuperar el territorio apropiado bajo el antiguo sistema de haciendas (Eherenreich, 1991). La Comuna sin embargo, empieza a perder fuerza política a finales de los setenta, cuando la economía pesquera crece rápidamente debido a la motorización. La Parroquia se organiza finalmente a mediados de los noventa y en un marco de progreso integra políticamente Salango al cantón más cercano. Con dinero del gobierno provincial construye un malecón completo con asfalto y luz eléctrica.

En la división entre «parroquianos» y «comuneros» los lazos de parentescos cumplen un rol importante y la definen como una estructura binaria. Dicha división se refleja en la economía de subsistencia: los «comuneros» son horticultores y los «parroquianos», pescadores. Se refleja también en la estructura de clases: los horticultores constituyen la clase social baja y los pescadores, la clase social alta. Sin embargo, el control de ciertos medios de producción tales como botes y redes, ocasiona diferenciaciones internas de clase entre los pescadores.

Las relaciones entre comuneros y pescadores tienen una contradicción permanente pero se han generado relaciones sociales específicas como mecanismos de equilibrio. Por ejemplo, existen alianzas matrimoniales entre pescadores y agricultores. Lo que a su vez ocasiona diferenciación ocupacional entre individuos del mismo grupo doméstico.

Dichas facciones políticas están sujetas también a crisis de carácter político, especialmente cuando fuerzas externas producen un mayor desajuste en las relaciones sociales existentes. Desde finales de los ochenta, un inversionista extranjero se fue convirtiendo gradualmente en el mayor terrateniente de la región. Apoyado por los miembros del cabildo, adquiere el título de comunero y compra una gran franja de territorio conocido como «Los Ostionales». A pesar de que «Los Ostionales» es una zona desolada, los habitantes locales tienen conocimiento de la existencia de una gran variedad de moluscos de fácil acceso en las áreas rocosas y otros productos marinos tales como pulpos y calamares.

Los parroquianos se alínean en contra del inversionista, denuncian la venta de la tierra como ilegal, y se aferran a sus raíces indígenas para incorporar una identidad precolombina: ManteñoHuancavilca. Con esta nueva identidad, justifican el reclamo de «la tierra ancestral» como un derecho natural. En oposición directa, La Comuna se alínea a favor del inversionista, declara las ventas de tierra como una transacción legal y renuncian a sus raíces indígenas para reforzar su identidad de montubios o descendientes de montubios. Con esta identidad, pueden justificar moralmente la venta de «la tierra ancestral». En esta área de la costa, los montubios por definición 
no son considerados indígenas ya que son el resultado de una continua inmigración desde el interior de las lomas hacia la costa ocurrida desde los últimos cientos de años (Eheremreich, 1991; Naranjo, 1980).

Los parroquianos con la ayuda de organizaciones indígenas nacionales y otros grupos políticos obtuvieron el apoyo mayoritario para apoderarse de La Comuna. El antiguo cabildo fue oficialmente desintegrado y uno nuevo fue instaurado bajo la dirección de un líder carismático. Cuando procesos electorales regulares se iniciaron para elegir nuevos líderes parroquiales, los comuneros desposeídos apoyaron a facciones familiares con cierto prestigio y los eligieron dentro del nuevo directorio parroquial. La recientemente electa Junta Parroquial nace en directa oposición a los intereses de la nueva Comuna, restableciéndose una vez más el equilibrio de poderes entre ambos grupos.

La existencia de facciones políticas con poderes duales probablemente es la continuación de un largo proceso histórico (Marcus, 1999). En Salango, en ausencia de una fuerza política integradora, una estructura binaria es la base sobre la cual la sociedad está organizada. Probablemente, el mismo patrón existía en el pasado, especialmente durante periodos en los cuales la autoridad política máxima estuvo centrada en otro lugar. Las dos estructuras de mayor fuerza hoy en día, La Parroquia y La Comuna, son organizaciones basadas en lazos de parentesco, y a través del tiempo alianzas de familias se han repartido el control de dichas organizaciones. Es evidente que la organización política refleja la dualidad de las formas de subsistencia y de los recursos básicos. En Salango, tierra y mar coexisten; horticultores y pescadores coexisten; comuneros y parroquianos coexisten inextricablemente. Ninguno se ha convertido en el grupo dominante.

\section{PROSPECCIÓN: ASENTAMIENTOS EN LA FRANJA COSTERA Y ASENTAMIENTOS EN EL INTERIOR}

La prospección se inicia en el año 2002 y tiene como objetivo determinar las diversas modalidades de ocupación territorial en la franja costera Puerto López (Ayampe). Dicho estudio nace frente a la necesidad de entender el entorno medioambiental de los principales asentamientos prehispánicos excavados anteriormente por el Programa de Antropología para el Ecuador (PAE) dirigido por Presley Norton (Currie, 1995; 2001; Lunniss, 2001; Mester, 1990; Norton et al., 1983) y para proponer nuevos modelos de asentamientos humanos a través del tiempo. Vislumbrar procesos de adquisición territorial, tomando en cuenta patrones de asentamiento ocurridos en un territorio con un alto grado de diversidad medioambiental, es indispensable para el entendimiento de la organización política, social y económica de sociedades prehispánicas en la costa de Manabí.

Durante la década de los setenta y ochenta y con el apoyo logístico/ financiero del PAE, se llevaron a cabo varias prospecciones arqueológicas (Damp, 1984; Allan \& Allan, 1989), que en su mayoría no han sido publicadas. La única referencia que podemos citar para nuestra región de interés es el artículo de Jonathan Damp (1984) sobre los sitios del Formativo Temprano en el valle del río Blanco. Los resultados de otras prospecciones se encuentran en los archivos del Centro de Investigaciones y del museo Salango. Debido a las diferencias de metodologías utilizadas en dichas investigaciones, a lo preliminar de los resultados y a las inconsistencias de los registros de campo, se tomó la decisión de corroborar en el terreno todos los sitios identificados anteriormente así como también, de expandir la zona de estudio. Uno de los objetivos secundarios de esta prospección es la elaboración de un mapa arqueológico de la zona que incluya un nuevo código de numeración de sitios asociados a mapas topográficos específicos siguiendo los nuevos lineamientos impuestos a nivel nacional por la oficina de Patrimonio Cultural.

Esta parte de la investigación se desarrolla en colaboración con la universidad de Neuchatel (Suiza). La prospección se realiza en una zona que comprende la franja litoral entre Puerto López (límite norte) y Ayampe (límite sur) y la red hidrográfica del río Blanco. Tiene una superficie total 
de $183 \mathrm{~km}^{2}$, de los cuales $170 \mathrm{~km}^{2}$ ya han sido prospectados. El área de estudio abarca los valles estrechos que desembocan directamente en el océano Pacífico (Pital, Salango, Chico, Puerto Rico, Tunas y cauce bajo del río Ayampe); así como también incluye la red hidrográfica del río Blanco con sus principales afluentes (Achiote, Tigre, Pólvora, Mocora, Tusas, Vaca, Cuchucho, Lodo, Bambas y Encantada).

La selección de esta microrregión y de la metodología de prospección siguió consideraciones climáticas, topográficas y medioambientales. En el sur de la provincia de Manabí, la cordillera costera (o de Chongón-Colonche) se acerca al océano Pacífico antes de tomar una dirección sureste hacia la ciudad de Guayaquil. El relieve pronunciado permite la condensación de los vientos marinos y la formación de garúa de verano. Esta particularidad regional favorece el crecimiento de una densa vegetación la cual se vuelve un obstáculo para la tarea de prospección. Nuestra estrategia de prospección por lo tanto, no fue uniforme en toda la región debido a problemas de accesibilidad, caminos vecinales y cauces de río.

A pesar de estas limitaciones, cada área de ocupación humana fue identificada a través de la presencia o ausencia de «vestigios culturales». Nuestro registro sistemático incluyó la descripción topográfica del entorno inmediato, la determinación de la cobertura vegetal y la determinación del uso moderno del terreno en cuestión. Así como también se efectuó un croquis tentativo de las áreas con vestigios culturales, un levantamiento topográfico básico y, la posición GPS para cada sitio establecido. Como fuente de datos suplementarios, se aprovechó también la presencia de barrancos profundos ubicados en los cauces de ríos secos. En estos barrancos, se abrieron largos perfiles estratigráficos que pusieron en evidencia una sucesión profunda de eventos culturales y naturales. Dicha profundidad de depósitos no se hubiera podido obtener con métodos tradicionales tales como pruebas de pala y pruebas con taladro.

Probablemente la parte más difícil de la tarea de prospección en esta zona fue la configuración del sitio arqueológico. Según Zadora-Río:

«[...] la mayoría de los arqueólogos establecen una diferencia de estatuto entre el hallazgo aislado y el sitio, teniendo a menudo dificultades en trazar la frontera que les separa [...]». (1986:12)

En nuestro caso seguimos la terminología señalada por Zadora-Río (1986) y se consideró que todo material cultural que no esté asociado directamente con un contexto arqueológico primario constituye un «hallazgo aislado». En un área con actividad fluvial constante, materiales arqueológicos en diversas ocasiones son depositados por fenómenos naturales o culturales en contextos secundarios. El contexto de deposición secundario de dichos materiales y la clara abrasión presente en ellos ayudó a determinarlos como «hallazgos aislados». Por otro lado, los «sitios arqueológicos» son todos aquellos en donde existe una clara asociación entre presencia de materiales culturales y el contexto de deposición. La presencia de depósitos culturales profundos, la existencia de estructuras domésticas, arquitectónicas y/o funerarias ayudaron también en dicha determinación.

Tomando en consideración los criterios arriba mencionados se ha definido hasta aquí 176 sitios arqueológicos y 9 hallazgos aislados. El área presenta una gran densidad de sitios (fig. 2). Las zonas grises señaladas en la figura 2 simbolizan los sitios de mayor extensión como por ejemplo: el sitio registrado en valle de El Triunfo, donde se observaron artefactos culturales en una superficie de más de $1 \mathrm{~km}$ a lo largo del cauce del río. Los triángulos oscuros en la misma área corresponden a sitios arqueológicos de menor tamaño, que probablemente representan aldeas secundarias.

Los resultados sugieren tres tipos de asentamientos en cuanto a su ubicación geográfica. Los sitios con una superficie considerablemente grande se encuentran en el fondo de los valles, sobre terrazas aluviales extensas. Este patrón es aparente en toda la franja costera de la zona bajo estudio. Damp (1984: 108-109) determina un patrón similar para el valle del río Blanco en donde amplios asentamientos humanos se establecen en las zonas bajas de los valles, casi siempre ubicados en la parte de atrás de los pueblos actuales (por ejemplo en Río Chico y Puerto 


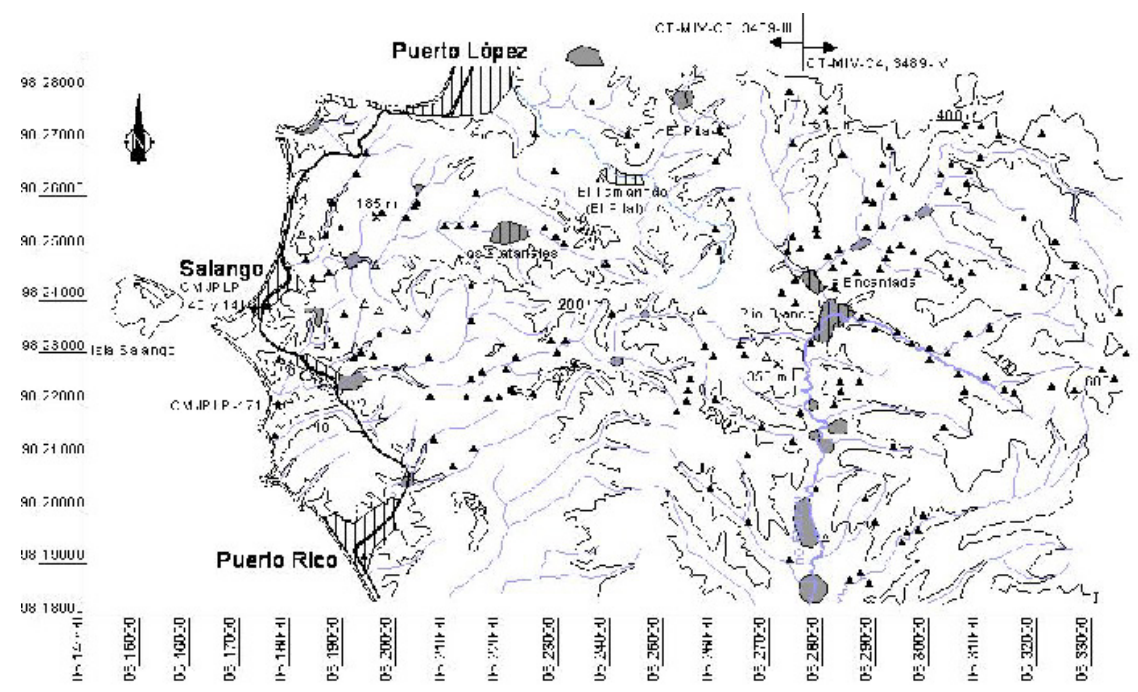

Figura 2 - Repartición de sitios en el área Puerto López-Ayampe (costa centro-sur, provincia de Manabí, Ecuador)

Mapa elaborado por Yann Graber

Rico). Por el otro lado, pequeñas aldeas se dispersan a lo largo de los cauces superiores. Sitios grandes también fueron localizados en las confluencias de ríos, particularmente en los estrechos valles tierra adentro. Finalmente, sitios de diversos tamaños fueron localizados en las lomas más altas, que hemos denominado «asentamientos de altura».

El patrón de asentamiento hasta aquí definido sugiere la presencia de sitios arqueológicos ubicados en los diversos medioambientes de la zona bajo estudio. Probablemente, dicha ubicación se correlaciona con la constante interacción de grupos humanos explotando diversos ecosistemas. Es interesante señalar además, la accesibilidad interregional que ciertas redes hidrográficas permitieron. Por ejemplo, la red hidrográfica del río Ayampe es una vía natural que conecta el litoral de Manabí, especialmente al sitio de López Viejo con la cuenca del Guayas en el interior. Finalmente, es indiscutible el rol económico que los sitios ubicados en la franja costera tuvieron en el intercambio de conchas marinas tal como lo sugieren varios investigadores (Martinez \& Martin, 2002; Luniss, 2001; Marcos, 1995; Currie, 1995; Norton, 1986; Murra, 1982).

Cronológicamente, todas las fases cerámicas de la costa ecuatoriana, desde Valdivia temprano — fase IV, siguiendo la cronología de Hill (1974) — hasta Manteño han sido identificadas. La asociación de sitios a fases cerámicas específicas fue posible a través de la presencia de diagnósticos tales como: figurines, elementos decorativos en la superficie de fragmentos cerámicos y características de la pasta. Determinaciones radio carbónicas sobre la antigüedad absoluta de los sitios será un objetivo futuro de nuestra investigación.

Los datos recuperados ponen a la luz también una gran variabilidad en cuanto a la función de los sitios prospectados. Por ejemplo, la ubicación geográfica del sitio N4C3-077, con acceso directo a un manantial de agua subterránea y la presencia de una alta densidad de material arqueológico de pasta fina y pintura iridiscente, sugiere una función ritual. El sitio está localizado en el valle del estero Lodo, cerca del pueblo actual de Río Blanco en un área donde el flujo de los ríos es estacional. La mencionada cerámica está concentrada en dos depósitos sucesivos de gran profundidad probablemente asociados con Engoroy temprano y tardío. El sitio abre la posibilidad de establecer comparaciones corológicas y cronológicas con otros sitios importantes de la región tales como Salango. 
El valle del río Salango presenta varios sitios domésticos localizados en las cimas de las terrazas o lomas. El sitio N4C3-040 contiene una serie de fogones de cocción y varias fosas de rechazo registradas en dos depósitos culturales separados por un depósito natural. La cerámica diagnóstica indica una ocupación u ocupaciones asociadas con la fase cultural Guangala y quizás fases culturales más tardías, por ejemplo Manteño temprano (ver sección de excavaciones). Dicho sitio - conjuntamente con otros registrados en las terrazas subtropicales — indica la dinámica interacción entre pobladores de la costa y el interior.

Finalmente, se registraron una serie de estructuras rectangulares de piedra o cimentaciones de estructuras - tierra adentro- en los valles de los ríos Blanco y La Moroca. Estructuras similares han sido registradas con anterioridad en los sitios de López Viejo (Currie, 1995), localizado en la franja costera, y Agua Blanca hacia el interior. Las estructuras encontradas por nuestra investigación sugieren que dicho material de construcción y patrón arquitectónico no se restringe únicamente a un solo medio ambiente.

\section{EXCAVACIONES: SITIOS DOMÉSTICOS Y SITIOS DE PRODUCCIÓN}

Los sitios hasta aquí investigados son tres: N4C3-170 (antiguamente denominado OMJ-PLP-170), localizado en la franja litoral; N4C3-040, localizado en las terrazas aluviales de régimen subtropical y; N4C3-035, localizado en terrazas aluviales de bosque seco.

El sitio N4C3-035 situado en una terraza con una altura de 38 m.s.n.m. está caracterizado por la presencia de un bosque seco. Sin embargo, habitantes del sector tuvieron acceso a otros medioambientes: el océano está ubicado a tan solo 500 metros hacia el oeste, y hacia el este se levanta una vegetación mucho más densa que forma parte del bosque húmedo subtropical. La cima de la terraza tiene un área aproximada de $300 \mathrm{~m}^{2}$, limitada en su parte oeste, este y norte por pendientes pronunciadas. Mientras la cima fue utilizada como zona habitacional, las pendientes sirvieron como zonas de enterramientos.

El sitio fue primero excavado a través de una operación de rescate cuando restos humanos fueron expuestos por la construcción (con maquinaria pesada) de un camino vecinal. Las labores de rescate incluyeron: excavación y recolección de restos culturales expuestos, reconstrucción del contexto cultural y determinación de la estratigrafía.

La reconstrucción del contexto cultural indicó que se trata de una tumba de pozo profundo con cámara lateral ubicada en la pendiente de la terraza. Originalmente, los restos humanos desarticulados de varios individuos fueron depositados en tres urnas funerarias asociadas con dos vasijas bruñidas negras y varios torteros. Las urnas fueron selladas por platos de base plana y superficie con ondulaciones en relieve, a los que Oyola-Courier (2000) clasifica como cómales. Los elementos decorativos del conjunto cerámico y el modo de enterramiento sugieren una posible asociación con la cultura manteña (ver Estrada, 1957; 1962). El análisis de la estratigrafía verificó la presencia de un solo depósito cultural por debajo de la capa vegetal de variada profundidad. Finalmente, se observó material cultural disperso en la parte sur de la cima de la terraza y quizás asociada con las tumbas.

Se tomó la decisión de continuar investigaciones en el sitio con una metodología de excavación más precisa. Las nuevas excavaciones en el área tuvieron los siguientes objetivos: verificar la existencia de otras tumbas, investigar la cima de la terraza y esclarecer una posible asociación entre los restos culturales de la cima y los restos humanos de la pendiente.

No se constataron más tumbas, pero las excavaciones sí corroboraron la presencia en la cima de un piso habitacional asociado a moldes poste, fosas de rechazo, áreas de combustión e innumerables fragmentos culturales (cerámica, lítica, concha, carbón, restos fáunicos, etc.). 
Análisis preliminares sugieren la existencia de por lo menos un área habitacional en donde una serie de actividades domésticas fueron ejecutadas.

Las estratigrafía en este sector también constató la existencia de un solo depósito cultural por debajo de la capa vegetal lo que sugiere que el área habitacional y el área de tumbas se encuentran en el mismo contexto deposicional y son por lo tanto contemporáneos. La presencia de elementos decorativos similares entre la cerámica de la cima de la terraza y aquellos encontrados en las tumba, refuerza esta interpretación.

Los análisis de restos osteológicos reconstruyeron 14 (MNI) individuos de variada edad (desde fetos hasta individuos de edad adulta media) y de ambos sexos (Jastremski, 2006). La muestra contiene individuos con enfermedades degenerativas de las coyunturas, osteoartritis de las vértebras, caries, infecciones en las órbitas, infecciones en los huesos, abscesos, caries, etc. lo que probablemente sugiere una población con un patrón de subsistencia variada (Jastremski, 2006: 71). Esta interpretación está reforzada por otras líneas de evidencia; los restos fáunicos contienen un alto contenido de recursos marinos y terrestres, y los restos líticos incluyen varias piedras de molienda tales como manos y metates.

El análisis osteológico propone también la existencia de especialidad ocupacional, cuya naturaleza todavía no ha sido clarificada. Jamstresky (2006) señala que la mayoría de las patologías para la población del sitio N4C3-035 indican individuos que estuvieron sujetos a actividades físicas diarias que les ocasionaron mucho estrés en las coyunturas de la espina dorsal, hombros, espalda, etc. Esto conjuntamente con la presencia de infecciones en los ojos y la evidencia del desarrollo de una musculatura robusta (evidenciada en las falanges y metatarsos de la muestra) proponen actividades relacionadas probablemente con la pesca y el buceo. Análisis adicionales serán realizados para corroborar o rechazar estas hipótesis.

El sitio N4C3-040 está localizado en la cima de una terraza aluvial en la parte sur del valle del río Salango y a 2 kilómetros del actual poblado de Salango. El área del sitio cubre aproximadamente $800 \mathrm{~m}^{2}$. Las excavaciones registraron una ocupación republicana y por debajo de ésta se registraron dos ocupaciones culturales (temprana y tardía) separadas por un depósito natural de tipo coluvial.

La ocupación prehistórica tardía contiene un depósito cultural profundo asociado con varias áreas de combustión, fosas de rechazo, moldes de poste y gran cantidad de restos culturales (cerámica, lítica, huesos fáunicos, concha, etc.). El análisis preliminar del material cultural indica la presencia de elementos asociados con ambos: cerámica Guangala tardía y cerámica Manteña. La posibilidad de que este sitio represente un sitio transicional entre el Periodo Regional e Integración será explorada en futuros análisis.

Por debajo de esta ocupación se registró un depósito natural asociado a piedras angulares de tamaño mediano y grande. Las rocas eran conglomerados amarillosos y es muy probable que este depósito coluvial represente un deslave con gran arrastre de rocas. Dicho evento natural selló rápidamente el depósito cultural inferior y seguramente es el resultado de una temporada de lluvias intensas.

El depósito cultural temprano contiene rasgos muy similares a los encontrados en el depósito cultural tardío, pero la cerámica presenta elementos diagnósticos asociados a la cultura Guangala con algunas influencias de Bahía. La cerámica presenta un grado de deterioro alto: está bastante erosionada y en muchos casos se está descascarando. Probablemente, los deslaves y agua subterránea han contribuido a la alta acidez y humedad de los suelos y son los causantes de la condición de la cerámica.

Debido a la presencia de gran cantidad de huesos de pescado y conchas que fueron encontrados en ambos depósitos culturales, es innegable el contacto entre los habitantes de este sitio y los habitantes de la franja costera. La base de subsistencia también incluyó una forma de agricultura ya que se recuperó gran cantidad de maíz carbonizado en ambos depósitos. 
Finalmente, el sitio N4C3-170 (antiguamente OMJPLP-170) localizado en el valle del río Chico es un sitio multicomponente. Basado en análisis cerámico se han reconocido los siguientes periodos de ocupación: Valdivia, Chorrera/Bahía, Guangala y Manteño (Oyola-Courier, 2000). Durante los periodos más tempranos, Río Chico fue principalmente un sitio doméstico, pero durante la época Manteña la función del sitio giro alrededor de la captura, procesamiento, almacenamiento y redistribución de Spondylus Calcifer exclusivamente.

Las excavaciones desenterraron evidencias de un estructura no doméstica de aproximadamente $50 \mathrm{~m}$ de largo por $20 \mathrm{~m}$ de ancho, construida paralela a la línea costera. Esta es la estructura no ceremonial de mayor tamaño que se ha registrado en la región. Las diversas áreas de actividad al interior de la estructura y material cultural asociado sugieren la existencia de un taller especializado con capacidad industrial.

En el interior de la estructura, se encontró una gran cantidad de fragmentos exhaustos de Spondylus. Patrones de las huellas de desgaste y de las fracturas indican que el borde de las conchas fue sistemáticamente extraído. Fosas de gran profundidad (hasta $2 \mathrm{~m}$ ) se encuentran distribuidas a lo largo de la estructura y probablemente fueron utilizadas para almacenar las conchas. Adicionalmente, varias zonas de combustión probablemente reflejan áreas de quema, resultado de la preparación y cocción de alimentos de los trabajadores.

El sitio de Salango es un sitio registrado históricamente como uno de los cuatro asentamientos que colectivamente formaron un gran señorío en el área (Norton et al., 1983). Es muy probable que durante la época Manteña, Salango y quizás López Viejo fueron los recipientes de las conchas procesadas provenientes de Río Chico. Ambos sitios han aportado evidencia de talleres especializados en la manufactura de cuentas y otros adornos personales (Currie, 1995; Norton et al., 1983).

\section{RESULTADOS PRELIMINARES}

Las investigaciones hasta aquí sugieren la presencia de sitios con una gran variabilidad en la utilización de recursos naturales lo que probablemente determinó la ubicación de los asentamientos. Es muy probable que el sitio N4C3-170 evidencie el desarrollo de una formación económica y política a nivel regional con propiedades internas interesantes, la cual formó parte de un comercio a larga distancia. Una de las características principales de la región es la existencia de sitios con sistemas de subsistencia bien desarrollados y probablemente autosuficientes desde épocas bastante tempranas. Dicho proceso culminó con la existencia de sitios especializados en la captura de conchas o en la manufactura de productos acabados.

Excavaciones futuras se concentrarán en el esclarecimiento de los patrones de explotación e intercambio de recursos naturales tanto a nivel local y regional. La prospección se concentrará en obtener información adicional para entender la integración entre sitios localizados en la franja litoral y sitios localizados hacia el interior. Es muy probable que el patrón de asentamiento de los sitios refleje la formación de unidades sociopolíticas que se fueron desarrollando e integrando a través del tiempo. Por otro lado, las investigaciones etnográficas otorgan no solamente información sobre la existencia de diversas subsistencias en el presente, sino también revelan cómo dichas subsistencias han determinado formaciones políticas y sociales. Varias de nuestras inquietudes científicas son siempre enfocadas desde dos direcciones: desde el presente hacia el pasado y desde el pasado hacia el presente. Este enfoque genera tensiones innovadoras en la investigación y genera nuevas direcciones para el campo de la antropología en general y en especial para la etnografía y la arqueología. 


\section{Agradecimientos}

Un gran reconocimiento a nuestros estudiantes de la Escuela de Campo del Departamento de Antropología de Florida Atlantic University, quienes a través de los años han contribuido con arduo trabajo a la realización de este proyecto. Muchas gracias a los varios colegas extranjeros y ecuatorianos, en especial a Angelo Constatine (quien descubrió y registro las estructuras en el valle del Río Blanco), por el apoyo continuo a nuestras investigaciones. Gracias al Instituto Nacional de Patrimonio Cultural y en especial a José Chancay, por las innumerables ideas compartidas con nosotros. Gracias al Centro de Investigaciones y Museo Salango por continuar adelante con su misión científica, y en especial a Patrick Gay, por su constante asistencia en el análisis de huesos fáunicos. Finalmente, mil gracias a la comunidad de Salango por ser los protagonistas principales de una historia cultural tan rica y compleja y por compartirla con nosotros.

\section{Referencias citadas}

ALLAN, P. \& ALLAN, R., 1989 - Prehistoric settlement distribution in the Río Salango valley; Salango, Ecuador. Informe del Programa de Antropología para el Ecuador en posesión del Centro de Investigaciones y Museo Salango.

CURRIE, E., 1995 - Prehistory of the Southern Manabí Coast, Ecuador: López Viejo; Oxford: BAR International Series 618.

CURRIE, E., 2001 - Manteño Ceremony and Symbolism: Mortuary practices and ritual activities at López Viejo, Manabí, Ecuador. In: Mortuary Practices and Ritual Associations: Shamanic elements in prehistoric funerary contexts in South America (Staller, J. \& Currie, E., eds.): 67-92; Oxford: BAR International Series 982.

DAMP, J., 1984 - Environmental Variation, Agriculture and Settlement Processes in Coastal Ecuador (3300-1500 B.C.). Current Anthropology, 25 (1): 106-111; Chicago: University of Chicago Press

EHERENREICH, J., 1991 - Antropología Política en el Ecuador: perspectivas desde las culturas indígenas; Quito: Abya-Yala.

ESTRADA, E., 1957 - Prehistoria de Manabí, Ecuador; Guayaquil: Publicaciones del Archivo Histórico del Guayas.

ESTRADA, E., 1962 - Arqueología de Manabí Central, Ecuador; Guayaquil: Publicaciones del Museo Víctor Emilio Estrada.

HILL, B., 1974 - A new chronology of the Valdivia ceramic complex. Nawpa Pacha Editions (10-12): 1-32; California: Berkeley Institute of Andean Studies.

JAMSTREMSKI, N., 2006 - Analisis of osteological remains from Salango, Ecuador with comparisons to four other coastal ecuadorian sites; Boca Raton: Department of Anthropology, Florida Atlantic University. Master Thesis.

LUNNISS, R., 2001 - Archaeology at Salango, Ecuador: an Engoroy ceremonial site on the south coast of Manabi; University College London. University Microfilms International, Ann Harbor, Michigan. PhD dissertation.

MARCOS, J., 1995 - El Mullu y el Pututo: la articulación de la ideología y el tráfico a larga distancia en la formación del estado Huancavilca. In: Primer encuentro de Investigadores de la Costa Ecuatoriana en Europa (Alvarez, S., Alvarez, A., Fauria, C. \& Marcos, J., eds.): 97-142; Quito: Abya-Yala.

MARCUS, G. E., 1999 - Critical Anthropology Now: Unexpected contexts, shifting constituencies, changing agendas; New Mexico: School of American Research. 
MARTíNEZ, V. \& MARTIN, A., 2002 - Spondylus Trade in Prehistoric South America: Establishing archaeological criteria to evaluate the movements of valuables. Paper presented at the 67th Annual Meeting of the Society for American Archaeologists, Denver, Colorado.

MESTER, A., 1990 - The pearl divers of Los Frailes: Archaeological and ethnohistorical explorations of sumptuary goods trade and cosmology in the North and Central Andes; University of Michigan, University Microfilms International, Ann Harbor, Michigan. PhD dissertation.

MURRA, J., 1982 - El Tráfico del Mullu en la Costa del Pacífico. In: Primer Simposio de Correlaciones Antropológicas Andino-Mesoamericano (Marcos, J. \& Norton, P., eds.): 265-274; Guayaquil: Escuela Superior Politécnica del Litoral.

NARANJO, M. F., 1980 - Etnicidad, Estructura Social y Poder en Manta: Occidente Ecuatoriano; Otavalo: Instituto Otavaleño de Antropología.

NORTON, P., 1986 - El Señorío de Salangome y la Liga de Mercaderes: El Cartel Spondylus-Balsa. Miscelánea Antropológica Ecuatoriana, 6: 131-144; Ecuador: Boletín de los Museos del Banco Central del Ecuador.

NORTON, P., LUNNISS, R. \& NAYLING, N., 1983 - Excavaciones en Salango, provincia de Manabí, Ecuador. Miscelánea Antropológica Ecuatoriana, 3: 9-72; Ecuador: Boletín de los Museos del Banco Central del Ecuador.

OYOLA-COURIER, M., 2000 - A preliminary investigation of ceramic styles and chronology at the Rio Chico site (OMJ-PLP-170), Manabí, Ecuador; Department of Anthropology, Florida Atlantic University, Boca Raton, Florida. Master Thesis.

POURRUT, P. \& GóMEZ, G., 1998 - El Ecuador al cruce de varias influencias climáticas: Una situación estratégica para el estudio del fenómeno del Niño. Bulletin de I'Institut Français d'Études Andines, 27 (3): 449-457; Lima.

VENTURA, M., 1997 - Etnografías Mínimas del Ecuador; Quito: Abya-Yala. Serie Pueblos del Ecuador 14.

ZADORA-RÍO, E., 1986 - La prospection archéologique et l'évolution de la notion de site. In: La prospection archéologique - paysage et peuplement (Ferdière, A. \& Zadora-Río, E., éds.); Paris: Éditions de la Maison des Sciences de l'Homme. Actes de la table ronde des 14 et 15 mai 1982. 